\title{
Métodos para Valoración del Perjuicio Estético Causado Por Cicatrices Faciales
}

\author{
Methods For Assessing Aesthetic Damage Caused By Facial Scars
}

\author{
Denisse Lagos Tissie ${ }^{1} \&$ Marcos Faúndes Pinto ${ }^{2}$
}

\begin{abstract}
LAGOS, T. D \& FAÚNDES, P. M. Métodos para valoración del perjuicio estético causado por cicatrices faciales. Int. J. Odontostomat., 15(2):532-537, 2021.

RESUMEN: Dentro del daño estético, las cicatrices son las secuelas más frecuentes. Al momento de su valoración médicolegal, hay que considerar las distintas formas en que se pueden presentar, además de su visibilidad a distancia. El objetivo de esta revisión es conocer los distintos métodos existentes usados para la valoración del perjuicio estético causado por cicatrices faciales. Se realizó una búsqueda bibliográfica en PubMed y Scielo, obteniéndose cuatro artículos atingentes al tema. Llama la atención la escasez de métodos estandarizados para su valoración, advirtiendo, además, que los pocos baremos existentes son para valorar el daño en el ámbito civil o laboral. Para estandarizar los criterios clínicos y medicolegales es recomendable el uso de métodos cuantitativos. Finalmente, al momento de evaluar las secuelas de las lesiones, es importante considerar no sólo el compromiso estético y funcional, sino también las implicancias biopsicosociales en la vida del individuo.
\end{abstract}

PALABRAS CLAVE: evaluación del daño, evaluación de cicatrices, daño facial, evaluación médicolegal.

\section{INTRODUCCIÓN}

La valoración del daño estético ha ido evolucionando, particularmente, por la importancia que adquieren la imagen y apariencia en la sociedad actual. Podemos definir el daño estético como "cualquier modificación que empeora la imagen de la persona. Es un perjuicio distinto del psicofísico que le sirve de sustrato y comprende tanto la dimensión estática como la dinámica". Las características fundamentales de este daño son: irregularidad o alteración corporal externa permanente y visible; desfiguración de la morfología del individuo de carácter peyorativo; produce un sufrimiento sentido como daño moral. El daño estético puede ser estático o dinámico (Instituto de Medicina Legal de Cataluña, 2014; Jalón et al., 2015):

- Estático: percibido a simple vista, afectando principalmente a la piel (cicatrices, quemaduras, pigmentaciones y deformaciones). También puede ser producido por pérdidas de segmentos corporales (amputaciones).

- Dinámico: afecta la actitud visible (trastornos en la marcha, la postura, la expresión y la mímica) o la actitud audible (afectación del habla).

Dentro de las secuelas, las más frecuentes y que provocan gran daño estético son las cicatrices. Al momento de la valoración médicolegal de éstas, hay que considerar que se pueden presentar de distintas formas: en buen estado, hipertróficas, discrómicas, tatuadas, con alopecias cicatriciales, retráctiles o queloideas. Por tanto, es muy importante realizar una descripción detallada de su apariencia, describiendo su situación, número, tamaño, forma, coloración, relieve, elasticidad y textura, comportamiento con estructuras anatómicas cercanas $\mathrm{y}$, sobre todo, su visibilidad, tanto a corta como a larga distancia. Cabe considerar que, a diferencia del daño físico general, el perjuicio estético genera controversias, toda vez que es un daño que, si bien se puede

\footnotetext{
${ }^{1}$ Odontóloga Forense, Servicio Médico Legal de Chile Región de Valparaíso, Valparaiso, Chile.

${ }^{2}$ Odontólogo Forense, Director Regional Servicio Médico Legal de Chile, Región de Valparaíso, Valparaiso, Chile.
} 
objetivar, involucra un importante componente de subjetividad por parte tanto del lesionado como del perito (Instituto de Medicina Legal de Cataluña).

El objetivo de esta revisión es conocer los distintos métodos que se usan en la actualidad para la valoración del daño estético causado por cicatrices faciales.

\section{METODOLOGÍA}

Se realizó una búsqueda en los motores PubMed y Scielo. En Scielo, se filtró la revisión para artículos con un máximo de 10 años de antigüedad con los conceptos "cicatrices faciales", "cicatriz rostro", "marca indeleble rostro" y "valoración cicatriz", obteniéndose un total de 20 artículos. Mientras que, en PubMed, se buscaron artículos publicados en los últimos 5 años, usando los conceptos "facial aesthetic AND damage", "aesthetic AND medico-legal evaluation" y "facial damage AND medico-legal evaluation", obteniéndose un total de 83 artículos. De estos 103 artículos, se eliminaron los duplicados, se realizó la lectura crítica de los abstract y se obtuvieron cuatro atingentes al tema de la revisión.

\section{RESULTADOS}

\section{Propuesta de método para valoración del perjui- cio estético por cicatrices.}

Rodes Lloret et al. (2013), realizaron la propuesta de un nuevo método para la valoración del perjuicio estético causado por cicatrices. Realizan una revisión y descripción breve de los métodos históricos usados para la valoración en todas partes del cuerpo y del rostro en particular. De los métodos antes mencionados, los autores comentan que solo tres son útiles en la valoración del daño estético causado por cicatrices en la cara (Rodes Lloret et al.):

- La "fórmula para el cálculo del daño estético causado por cicatrices en cualquier parte del cuerpo" de Bermúdez, valora variables como la longitud y anchura de la cicatriz, la localización de la misma, estableciendo para ello cinco zonas y ordenándolas de mayor a menor visibilidad, su relación con pliegues y arrugas y características de la cicatriz. Este autor añade en la valoración factores como el sexo, la edad y el estado anterior.

- El "método de las distancias del daño estético para cicatrices en la cara" de Rouge et al, que establece criterios descriptivos, valorándose tanto la localización de la cicatriz como sus características, además de un criterio normativo (distancia a la que se percibe la cicatriz). Una vez valorada la cicatriz, se traslada a la tabla correspondiente que recoge siete grados de daño estético.

- El "coeficiente del daño estético para cicatrices en la cara" se utiliza para evaluar el daño estético que ocasionan las cicatrices localizadas en la cara. Multiplica el coeficiente de localización (máximo 10 puntos) por el coeficiente de identificación (máximo 10 puntos), con lo que el resultado oscilará entre 0 y 100 puntos.

Los autores proponen un nuevo método aplicable cuando existe la presencia de solo una cicatriz. Se valoran tres parámetros: zona del cuerpo donde está la cicatriz, longitud de la misma y distancia a la cual se ve. Estos parámetros se valoran en un $70 \%, 10 \%$ y $20 \%$, respectivamente. La puntuación resultante se lleva a una segunda tabla, la que valora dos parámetros (incrementando o reduciendo la puntuación obtenida): deformidad de la cicatriz (no deformidad, ligera deformidad, moderada deformidad, gran deformidad, intensa deformidad) y color de la cicatriz (no destaca, destaca ligeramente, destaca moderadamente, destaca mucho, destaca intensamente) (Rodes Lloret et al.) (Fig. 1).

\section{Valoración del perjuicio estético en el rostro en la clínica médico forense.}

Barboza Quirós (2015) analizó una serie de casos penales, evaluando los criterios médicolegales considerados al evaluar perjuicio estético en el rostro. El autor comenta que, en su país, se evalúan tres componentes: alteración de la armonía o simetría del rostro de carácter llamativo, visibilidad y permanencia y si produce deformidad sin llegar a desfigurar (Barboza Quirós).

El autor describe dos métodos para valorar el perjuicio estético en el rostro: descriptivo y cualitativo. El método descriptivo básicamente consiste en describir la alteración estética lo más detalladamente posible, tomando en cuenta todos los aspectos tanto morfológicos como de funcionalidad. El método cualitativo busca establecer una calificación de la importancia del daño estético, la cual posteriormente se correlaciona con una escala gradual calificativa o nu- 


\begin{tabular}{|c|c|}
\hline Parámetros para valorar & $\begin{array}{c}\text { Puntuación (máximo: } \\
100 \text { puntos) }\end{array}$ \\
\hline ZONA DEL CUERPO & 70 PUNTOS \\
\hline Planta del pie, axila & 1 punto \\
\hline Glúteo, área genital & $S$ puntos \\
\hline Dorso del pie & 10 puntos \\
\hline Tórax, abdomen, espalda & 25 puntos \\
\hline Brazo, muslo & 30 puntos \\
\hline Antebrazo, pierna & 40 puntos \\
\hline Mano & 50 puntos \\
\hline Cuello & 60 puntos \\
\hline Cara & 70 puntos \\
\hline LONGITUD EN CM* & 10 PUNTOS \\
\hline Hasta el noveno $\mathrm{cm}$ & 1 punto cada $\mathrm{cm}$ \\
\hline $100 \mathrm{mb} \mathrm{cm}$ & 10 puntos \\
\hline DISTANCIA A LA QUE SE VE & 20 PUNTOS \\
\hline $\begin{array}{l}50 \mathrm{~cm} \\
1 \mathrm{~m}\end{array}$ & $\begin{array}{l}3 \text { puntos } \\
8 \text { puntos }\end{array}$ \\
\hline $2 \mathrm{~m}$ & 10 puntos \\
\hline $3 \mathrm{~m}$ & 15 puntos \\
\hline $4 m$ & 17 puntos \\
\hline 5 o más metros & 20 puntos \\
\hline TOTAL & \\
\hline
\end{tabular}

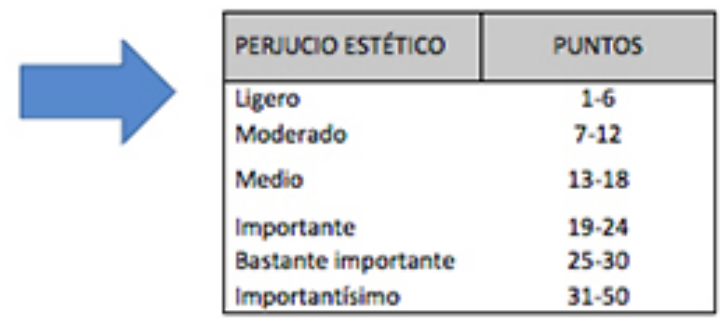

\begin{tabular}{|c|c|c|c|c|c|}
\hline Deformidad/color & $\begin{array}{c}\text { No } \\
\text { deformidad }\end{array}$ & $\begin{array}{c}\text { Uigera } \\
\text { deformidad }\end{array}$ & $\begin{array}{l}\text { Moderada } \\
\text { deformidad }\end{array}$ & \begin{tabular}{|c|} 
Gran \\
deformidad
\end{tabular} & $\begin{array}{c}\text { Intensa } \\
\text { deformidad }\end{array}$ \\
\hline No destaca & $\begin{array}{c}\text { Reducción } \\
90 \% \\
\end{array}$ & $\begin{array}{c}\text { Reducción } \\
60 \%\end{array}$ & $\begin{array}{c}\text { Reducción } \\
\text { so\% }\end{array}$ & - & $\begin{array}{c}\text { Incremento } \\
5 \%\end{array}$ \\
\hline Destaca ligeramente & $\begin{array}{c}\text { Reducción } \\
60 \%\end{array}$ & $\begin{array}{c}\text { Reducción } \\
\text { sox }\end{array}$ & - & $\begin{array}{c}\text { Incremento } \\
5 \%\end{array}$ & $\begin{array}{c}\text { Incremento } \\
10 \%\end{array}$ \\
\hline $\begin{array}{l}\text { Destaca } \\
\text { moderadamente }\end{array}$ & $\begin{array}{c}\text { Reducción } \\
50 \%\end{array}$ & ( & $\begin{array}{c}\text { Incremento } \\
5 \%\end{array}$ & $\begin{array}{c}\text { Incremento } \\
10 \% \\
\end{array}$ & $\begin{array}{c}\text { Incremento } \\
15 \%\end{array}$ \\
\hline Destaca mucho & $\cdot$ & $\begin{array}{c}\text { Incremento } \\
5 \% \\
\end{array}$ & $\begin{array}{c}\text { Incremento } \\
10 \% \\
\end{array}$ & \begin{tabular}{|c|} 
Incremento \\
$15 \%$ \\
\end{tabular} & $\begin{array}{c}\text { Incremento } \\
20 \% \\
\end{array}$ \\
\hline Destaca intensamente & $\begin{array}{c}\text { Incremento } \\
5 \%\end{array}$ & $\begin{array}{c}\text { Incremento } \\
10 \%\end{array}$ & $\begin{array}{c}\text { Incremento } \\
15 \%\end{array}$ & $\begin{array}{c}\text { Incremento } \\
20 \% \\
\end{array}$ & $\begin{array}{c}\text { Incremento } \\
25 \%\end{array}$ \\
\hline
\end{tabular}

mérica. Esta calificación se puede hacer de dos maneras: de una forma empírica apoyada en el método descriptivo y una analítica la cual utiliza fórmulas matemáticas cuyo objetivo es cuantificar el daño en términos numéricos que luego permita ubicar dicho daño en alguna de las categorías establecidas en las escalas de gradación (Barboza Quirós).

El autor refiere que, en su país, no está bien establecido el tema en relación a la valoración del perjuicio estético. Menciona que su Código de Trabajo establece un rango de porcentaje de pérdida de la capacidad general para las cicatrices del rostro que ocasionan desfiguración facial y que alteran la presentación física personal. Mientras que, desde el punto de vista del derecho penal, no existen baremos o protocolos que faciliten una estandarización en las valoraciones médico legales (Barboza Quirós).
El autor propone un método de valoración tomando como base criterios descriptivos y analíticos. El primer parámetro a tomar en cuenta es la visibilidad tanto a distancia de conversación como distancia social, e incluye un apartado para las cicatrices y otro para asimetrías. El segundo parámetro corresponde a la ubicación, para lo cual divide el rostro por zonas La zona A corresponde a las zonas más visibles del rostro; la zona $B$, a la región frontal, pómulos, mejillas y mentón; y la zona $C$ (sienes y región maxilar inferior), la zona menos visible del rostro. El siguiente parámetro es aplicable únicamente a las cicatrices, pues considera si éstas son viciosas y les asigna un puntaje de acuerdo a ello. Otros parámetros a tomar en cuenta son el área afectada, la que resulta de obtener las medidas de longitud y anchura, y la afectación de la mímica facial. Una vez que se tiene el puntaje de todos los parámetros expuestos se realiza la 
Tabla I. Método de valoración medicolegal del daño estético facial propuesto por Barboza Quirós (2015).

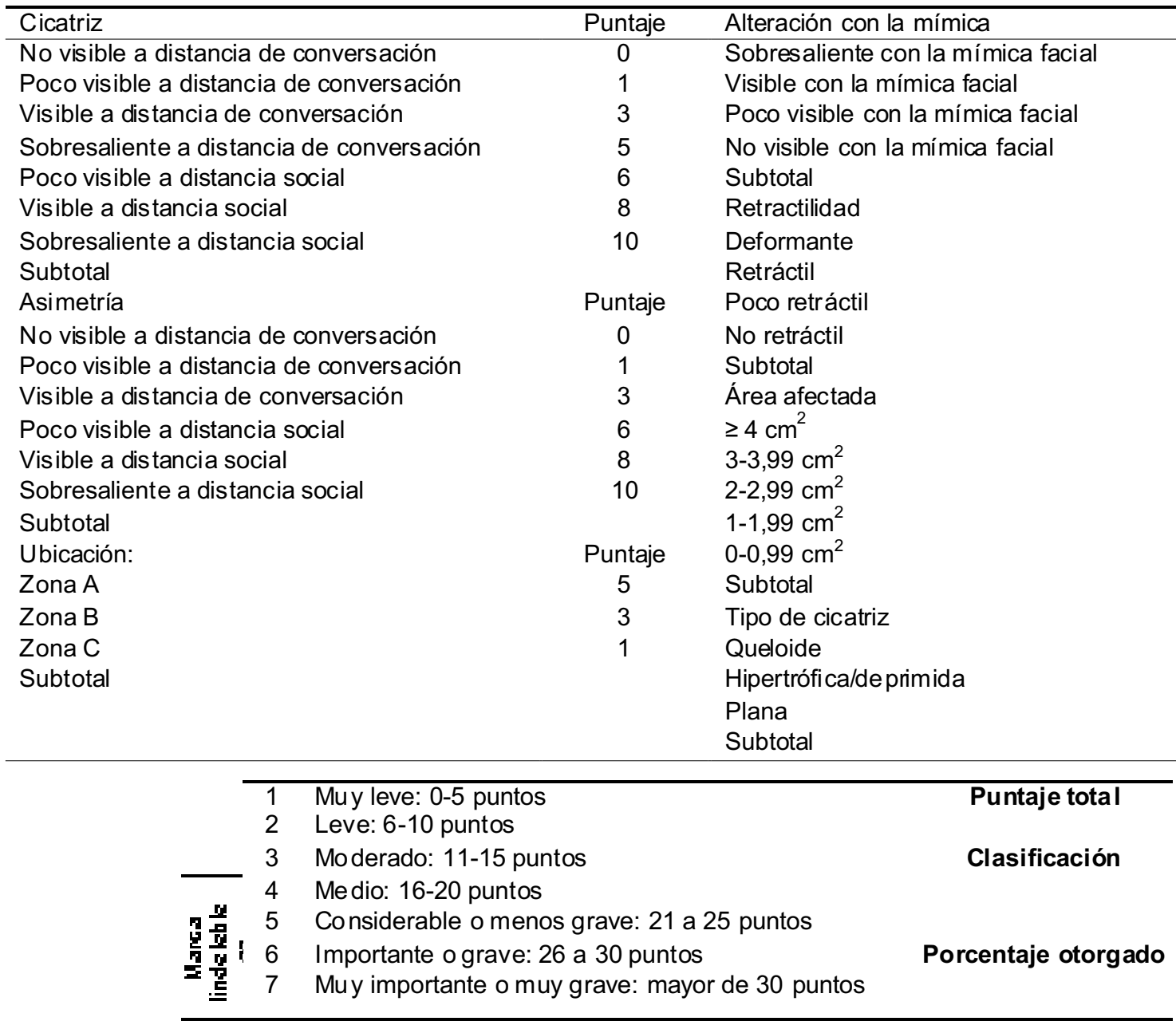

sumatoria de los mismos y el puntaje global se ubicaría en alguna de las categorías de una escala cualitativa (Barboza Quirós) (Tabla I).

\section{Correlación de análisis de expertos en la valora- ción de cicatrices faciales.}

Russo et al. (2019) realizaron un estudio en el que presentan 60 fotografías con daños estéticos estabilizados a 16 evaluadores expertos. Éstos debían cuantificar exclusivamente el componente objetivo de la discapacidad estética basados en los lineamientos de las "National Guidelines for the assessment of personal impairment". Los resultados mostraron que los evaluadores fueron más consistentes en la cuantificación del daño en sujetos masculinos y en mayores de 50 años, así como en la evaluación de daños extensos por sobre los de gravedad leve. La evaluación de deficiencias del área facial tuvo un mayor grado de concordancia respecto a los localizados en las regiones de cabeza y cuello. En general, se aprecia un alto grado de concordancia general en la habilidad de los profesionales medicolegales para formular un juicio complejo de la manera más objetiva posible. Los autores, en su discusión, comentan lo previsible de la dificultad de establecer el daño facial en mujeres, pues parece ser que para el sexo femenino es más importante la estética facial; además, lo previsible de la dificultad de establecer el daño en jóvenes y/o niños, pues resulta complejo predecir la importancia real de la deformidad estética en la vida del individuo, en sus relaciones sociales o en su vida laboral. Asimismo, comentan que hay mayor concordancia para calificar la gravedad de las cicatrices dejadas por quemaduras por sobre las iatrogénicas o de otra etiología, pues, generalmente, las primeras conllevan daños de mayor gravedad (Russo et al.).

Recomiendan que la valoración no sea solo en base a los parámetros objetivos, sino en lo expresivo, 
psicológico y las características sociales del individuo. Los criterios sugeridos por los autores son: estabilización de la lesión, características objetivas de la lesión, posibles deficiencias funcionales concomitantes, consecuencia en la vida relacional y su posibilidad de recuperación mediante cirugía (Russo et al.).

\section{Software para la evaluación cuantitativa de daño estético.}

Si bien este último artículo no está enfocado sólo en cicatrices faciales, es de interés su inclusión, pues es el único que incorpora la tecnología en la evaluación médicolegal de lesiones y es bien sabido que los softwares en la práctica clínica pueden disminuir la cantidad de tiempo dedicado al análisis y abaratar el costo de los mismos, lo que impacta positivamente en la eficiencia del servicio.

El estudio de Sakaguti et al. (2019) permitió la construcción de un software de libre acceso para la evaluación de daño estético corporal. Los autores se basan en un sistema de evaluación de daño estético desarrollado el año 2010 (método AIPE). Este método evalúa la severidad del deterioro estético y una posible deformidad. El método consta de cuatro tablas, la primera de las cuales contiene cinco preguntas. Para la evaluación de daños estéticos, este enfoque emplea cinco niveles de percepción para el observador, comenzando con la prueba de daño y terminando con el tipo de emoción que causa. A medida que avanza cada nivel, el grado de daño estético aumenta. El método propone las siguientes preguntas (Sakaguti et al.):
1. ¿Es posible percibir la alteración en la imagen de la persona?

2. ¿Nuestra vista tiende a centrarse específicamente en ese defecto?

3. Cuando recordamos al afectado, ¿lo describimos en torno a su daño estético?

4. ¿El daño estético causa alguna emoción en el afectado (tristeza, inseguridad, etc.)?

5. ¿La lesión afecta sus relaciones familiares o sociales?

Según los autores, el llenado en la plataforma es intuitivo e interactivo, y el texto puede ser personalizado por el usuario. El programa recopila la información y transforma el informe en PDF, guardando todas las evaluaciones. La información está encriptada para mayor seguridad y confidencialidad del paciente (Sakaguti et al.). El software está disponible en el sitio web en https://www.aestheticdamage.com (Fig. 2).

\section{DISCUSIÓN}

La valoración del perjuicio estético, especialmente el causado por cicatrices en la zona facial, está sujeto a la subjetividad, tanto del evaluador como del afectado. A esto hay que sumar que el concepto de "estética" no es igual para todos y puede ir variando en función de la edad de la persona, su ocupación, sus relaciones sociales, entorno, etc. Si bien esta realidad es conocida por todos los profesionales forenses que evalúan cicatrices en esta zona del cuerpo, llama la atención la escasez de métodos estandarizados para

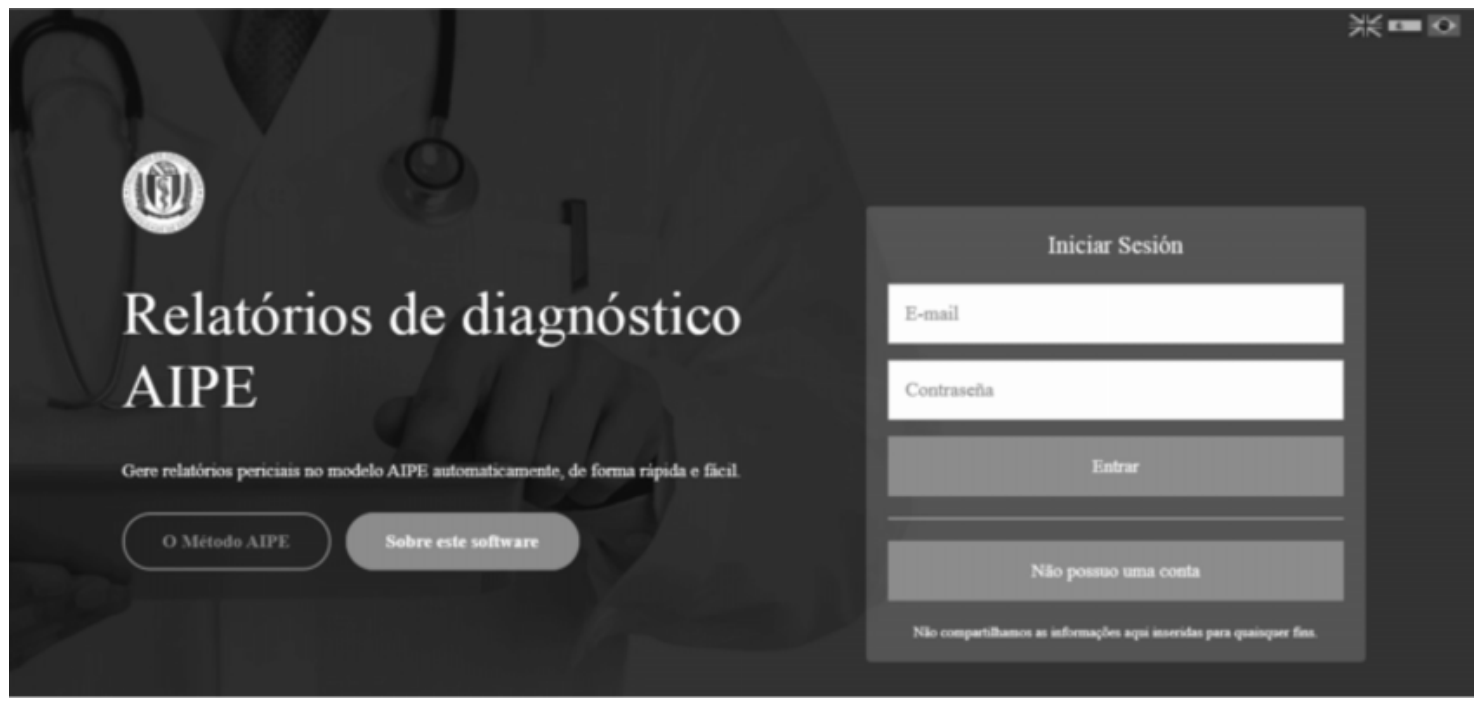

Fig. 2. Software AIPE. 
su valoración, advirtiendo, además, que los pocos baremos existentes son para valorar el daño en el ámbito civil o laboral y no en materia penal.

Los autores de los artículos expuestos en esta revisión parecen coincidir en que, para estandarizar los criterios clínicos y medicolegales, es recomendable el uso de métodos cuantitativos (baremos), aceptados y conocidos no solo por el personal que realiza la valoración, sino también por las autoridades encargadas de la administración de justicia. Por otra parte, los autores hacen énfasis que, al momento de evaluar las secuelas de las lesiones, es importante considerar no solo el compromiso estético y funcional, sino también las implicancias biopsicosociales en la vida del individuo.

El caso particular de Chile no es ajeno a la realidad antes expuesta: no teniendo normativa específica para la valoración de cicatrices faciales, la valoración se hace de manera cualitativa, según el tiempo de incapacidad laboral o el tiempo que demoren en reparar las lesiones, como está tipificado en el Código Penal: lesiones gravísimas, lesiones simplemente graves, lesiones menos graves y lesiones leves. La calificación de lesión "gravísima" no responde a un criterio clínico, sino judicial, y el juez las sanciona así cuando producto de las lesiones "queda el ofendido demente, inútil para el trabajo, impotente, impedido de algún miembro importante o notablemente deforme" (Código Penal, 2020). Como es sabido, los informes medicolegales son emitidos a petición de una autoridad judicial. En ellos, habitualmente, el fiscal solicita al perito referirse a la naturaleza de la lesión, tiempo que demora en sanar, mecanismo causal. Sin embargo, además de contestar estas preguntas, y cuando las lesiones son en la cara o rostro de la persona, el fiscal puede solicitar la opinión experta en cuanto al compromiso estético de la misma: si el perito lo considera así, lo pondrá en su informe y, muy probablemente, el juez valorará la lesión de distinta manera (como lesión gravísima, por ejemplo). Esta última situación parece ser la manera que, en nuestra legislación, podemos valorar el perjuicio estético en el rostro de manera distinta a las lesiones de otras partes del cuerpo.

LAGOS, T. D \& FAÚNDES, P. M. Methods for assessing aesthetic damage caused by facial scars. Int. J. Odontostomat., 15(2):532-537, 2021.

ABSTRACT: In relating to facial aesthetic damage, scars are the most frequently observed. When these scars are assessed for medico-legal purpose, it is necessary to consider the different ways in which they can be presented, and their visibility from distance. This review has as objective to evaluate the different existing methods used for the assessment of cosmetic damage caused by facial scars. The bibliographic search was carried out in PubMed and Scielo, where four articles related to the topic were found. The shortage of standardized methods for their assessment is striking, in addition, draws attention that the few existing scales are used to assess the damage in civil and labour affairs. To standardize clinical and medico-legal criteria, the use of quantitative methods is recommended. Finally, to evaluate the result of the injuries, it is important to consider not only the aesthetic and functional consequences, but also the biopsychosocial implications in the life of an individual.

KEY WORDS: Damage assessment, scar evaluation, facial damage, medico-legal evaluation.

\section{REFERENCIAS BIBLIOGRÁFICAS}

Barboza Quirós, M. Análisis de los criterios médico legales para la valoración del perjuicio estético en el rostro en la clínica médico forense: estudio de casos penales en el año 2011. Med. Leg. Costa Rica, 32(1):23-39, 2015.

Código Penal. Diario Oficial de la República de Chile. 4 de marzo del 2020. Santiago de Chile, Diario Oficial de la República de Chile, 2020.

Instituto de Medicina Legal de Cataluña. Controversias en Valoración del Daño Corporal. Capítulo VII: Valoración del Daño Estético: Criterios Evaluadores. Barcelona, Generalitat de Catalunya. Centre d'Estudis Jurídics i Formació Especialitzada, Institut de Medicina Legal de Catalunya, 2014.

Jalón, V. B.; Seco, M. D. A. \& Espinosa, C. G. El perjuicio estético en el nuevo baremo. Rev. Esp. Med. Legal, 41(4):208-11, 2015.

Rodes Lloret, F.; Sánchez Navas, L. D.; Borrás Rocher, F.; Pastor Bravo, M. \& Cañete de Silva, Z. Propuesta de un método para la valoración médico legal del perjuicio estético por cicatrices. Cuad. Med. Forense, 19(1-2):13-9, 2013.

Russo, M.; Bolcato, M.; Sabadin, V. \& Aprile, A. The medico-legal assessment of aesthetic damage. A correlation analysis between experts and an operative proposal. Leg. Med., 40:47-53, 2019.

Sakaguti, N. M.; Fernandes, M. M.; Mazzilli, L. E. N.; Plana, J. A. C.; Bouchardet, F. C. H. \& Nogueira de Oliveira, R. Development of a computer-aided design software for the quantitative evaluation of aesthetic damage. PLoS One, 14(12):e0226322, 2019.

Dirección para correspondencia:

Denisse Lagos Tissie

Odontóloga Forense

Servicio Médico Legal de Chile

Región de Valparaíso

Valparaíso

CHILE

E-mail: dlagos@sml.gob.cl 